\title{
ARTIFICIAL HEART VALVE INSPECTION USING EDDY CURRENT TECHNIQUES
}

This paper discusses about minimally invasive techniques to detect the single leg fracture in special type of artificial heart valve called Bjork Shiley Convexo - Concave (BSCC). The fracture affects the outlet strut of these artificial heart valve and it is needed to detect it. For this purpose the electromagnetic methods based on eddy current testing were used. The coils are excited in the selected range of the frequencies for identification of the fractures in the outlet strut. The fractures occurring in BSCC heart valve and their numerical simulations are described. The results obtained with a prototype setup are presented and they demonstrate the usefulness of described techniques for detection of the outlet strut fractures in BSCC artificial heart valve.

\section{Introduction}

Dysfunction of the heart valves is a common complication after heart valve diseases. When the symptoms become intolerable for a normal lifestyle, the normal course of action is to replace the damaged valve with an artificial one. These prosthetic valves are usually made from metal and are called - mechanical ones or they are made from biological tissue. The special type of mechanical heart valve prostheses is a Bjork-Shiley Convexo Concave (BSCC) heart valve which was introduced in 1979. BSCC heart valve has been used to replace the aortic or mitral valves and the design was developed in order to improve the hemodynamic and to reduce the risk of thromboembolism. This model of the heart valve became the subject of a lawsuit because it was shown to malfunction, usually fatally, in a number of cases. During the eight years that the valve was on the market, approximately 86000 valves were implanted in to patients. Six hundred and fifty valves experienced outlet strut fractures. As a result, the opportunity to evaluate the state of the BSCC heart valve, a non-destructive test of each individual valve

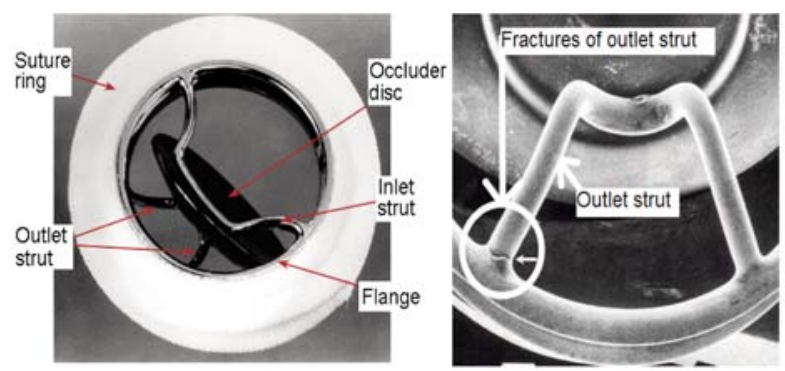

Fig.1 Typical design of the BSCC heart valve with fracture at the outlet strut [1] may help to detect the fractures presented in outlet strut and so precede the damage of the valve.

The BSCC valves have a carbon occluder disc held in place by two metallic struts. Of the two struts, the inlet strut is integral to the valve suture ring, while the other strut called the outlet strut is welded to the suture ring, Fig. 1 [3]. Artificial heart valves are engineered devices which must be designed to survive more than 40 years of operation. Furthermore, the process of cyclic slamming open and shut of the occluder disc during heart function subjects the valve to percussive impact stress, which can cause fatigue failure. These failures have been observed at the outlet strut flange junction near or at the weld. The failure of both struts allows for dislodgement of the occluder disc and embolization of the disc. A dual strut failure results in abrupt onset of dyspnea, loss of consciousness, or cardiovascular collapse due to embolization of the disc and acute severe valvular regurgitation. Patient with strut fracture of aortic prosthesis dies in minutes, but those with strut fracture of mitral prosthesis may survive long enough to undergo valve replacement. Several types of mechanical BSCC heart valve failure have been reported in literature

1. An outlet strut fracture (OSF), fracture of both legs of the outlet strut, the strut is completely separated from the flange

2. A single leg fracture (SLF), fracture of the one leg of the outlet strut, both ends grate against each other

3. A single leg separation (SLS), fracture of the one leg of the outlet strut, both ends of the fractured strut are separated, Fig. 1.

Although the function of the valve can continue under these conditions in points 2,3 , it increases the stress concentration on the intact end of the welded strut and it is not clear how long the other end of the strut can remain intact [1]. The period of the

\footnotetext{
* Tatiana Strapacova, Klara Capova, Ladislav Janousek

Department of Electromagnetic and Biomedical Engineering, Faculty of Electrical Engineering, University of Zilina, Slovakia,

E- mail: strapacova@fel.uniza.sk
} 
time between the first and the second fractures is highly variable Due to this fact there is a considerable interest, therefore, in development of methods for assessing the state of the valve in general condition of the outlet strut weld. In the present paper we propose electromagnetic techniques - eddy current testing (ECT) for nondestructive examination of the outlet strut of BSCC heart valve. To investigate the given problem, it is convenient to realize it with the aid of numerical simulation techniques.

\section{Eddy Current Testing - ECT}

Non-destructive testing (NDT) of conductive materials requires high reliability to detect cracks and defects in advance. ECT is one of the non-destructive techniques often used to detect them. In EC testing, the probe coil excited with alternating current is placed on an electrically conductive material (metallic biomaterial), eddy current are induced in the material. Presents of inhomogeneities, defect, variations in electrical conductivity, magnetic permeability, and geometry of the material, and lift- off, etc. disturb the eddy current flow and in turn, alter the impedance of the pick up coil. The change in impedance is usually measured and correlated with defect dimension or the causes producing it. In order to detect the defects sensitively, high frequency exciting currents or appropriate lift-off have to be applied. This method is applicable for surface or subsurface flaw detection because of significant decrease in magnetic flux and eddy current density with depth. The depth of penetration of eddy current is limited by skin-effect, which depends on operating frequency, material conductivity, and permeability [2] The theoretical background of the ECT was given e.g. in [3] and [4].

\section{Numerical Evaluation Using ECT - Model Configuration}

Possibilities of the eddy current inspection of BSCC are investigated in the paper using numerical means. The commercially available software OPERA-3D based on finite element method is utilized for the purpose.

The problem deals with absolute coil type, placed above an outlet strut of BSCC heart valve replacement. The probe coil with dimensions, Fig. 2a, has axis-symmetric shape and has 10 turns. It is supplied with current density $J=1 \mathrm{~A} / \mathrm{mm}^{2}$. Simulations were performed with various settings of lift-off $s_{1}=1 \mathrm{~mm}, s_{2}=2 \mathrm{~mm}$, $s_{3}=3 \mathrm{~mm}, s_{4}=5 \mathrm{~mm}$ (analogy to real motion of outlet strut in the real human body). Frequency of the driving harmonic coil signal was also changed from $10 \mathrm{kHz}$ up to $500 \mathrm{kHz}$.

Properties of the investigated material, dimensions and electromagnetic parameters of the heart valve replacement were set according to the real dimensions and electromagnetic parameters of the heart valve replacements. The materials commonly used for the conductive heart valves replacement are Stainless steel 316L, CoCr -F 75, F 90 alloys and Titanium alloy -Ti-6Al-4V. For our simulation the BSCC heart valve was made from titanium alloy Ti-
$6 \mathrm{Al}-4 \mathrm{~V}$ thus the conductivity was $\sigma=1.4 .10^{6}[\mathrm{~S} / \mathrm{m}]$ and relative permeability $\mu_{r}=1$.
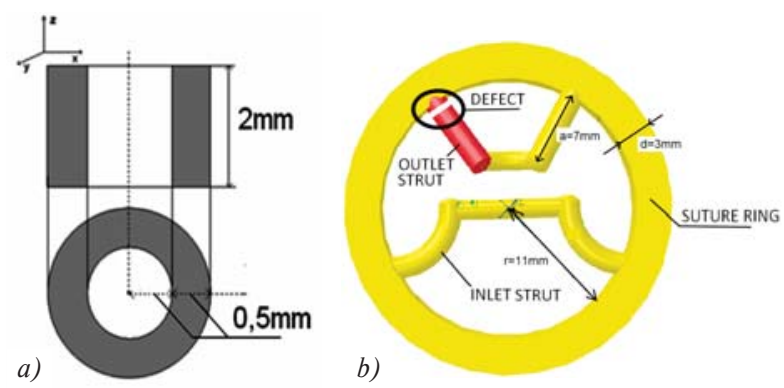

Fig. 2 a) Dimension of the coil, b) Dimension of the BSCC heart valve

The defect was localized on the one end of the outlet strut, Fig. 2b. The defect width was $w=0.1 \mathrm{~mm}$, the defect depths were changed during inspection. The depths of defect were following $r_{1}=0.1 \mathrm{~mm}, r_{2}=0.3 \mathrm{~mm}, r_{3}=0.5 \mathrm{~mm}, r_{4}=0.7 \mathrm{~mm}, r_{5}=0.9 \mathrm{~mm}$. The defects occurs in direction of the outlet strut diameter. The diameter of the outlet strut was set $d=2 \mathrm{~mm}$. Material defect has zero conductivity $\sigma=0[\mathrm{~S} / \mathrm{m}]$. The dimensions, orientation and depth of the defect were set up according to the real dimensions and depth of defect that affects outlet strut of BSCC heart valve. Such types of the defect can be presented as fatigue cracks.

Numerical simulations of the developed model are carried out to calculate the probe response signal. The finite element mesh of the simulated problem has approximately 3 million elements of two types, linear and quadratic ones. The following sections present the results.

\section{Numerical Evaluation Using ECT with Helmholtz Coils - Model Configuration}

The ECT with Helmholtz coils based approach was considered. The problem deal with Helmholtz coils placed on each side of the heart valve. These coils are configured to ensure the uniformity of the generated magnetic field. The excitation coils with dimensions, Fig. 3, are supplied with current density $J=1 \mathrm{~A} / \mathrm{mm}^{2}$. Frequency of the driving harmonic coils signal was changed from $10 \mathrm{kHz}$ up to $500 \mathrm{kHz}$. The EM field excited by Helmholtz coils was oriented in parallel direction to the suture ring. The detection coil was positioned in distance lift-off $=1 \mathrm{~mm}$ above the outlet strut where the crack arises. The coil detect the perturbation of the generated field due to defect. The voltage induced in the detection coil was evaluated and the results are displayed on the Fig. 7.

\section{Numerical Simulation Results the ECT Results}

The material object (BSCC heart valve) and the coil with given dimensions were used for numerical simulation of eddy current evaluation. The depth of defect, lift-off and the frequency of the 


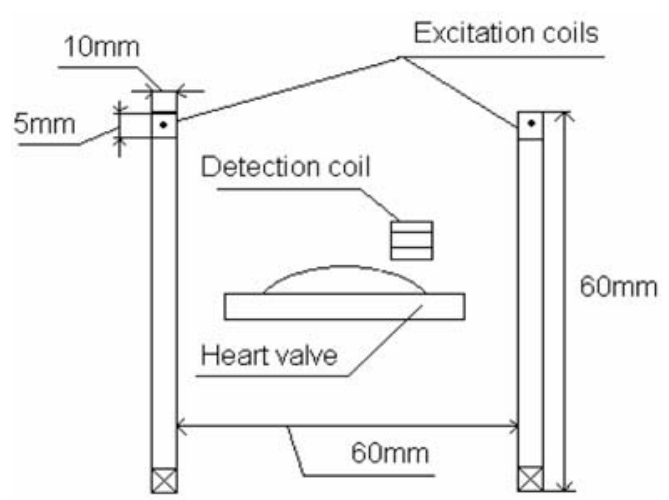

Fig. 3 Geometry of the electromagnetic system

driving signal were changed during the simulations. It represents 124 simulations realized. The $\left|\Delta Z_{m}\right|$ represents the difference amplitude impedance signal $\mathrm{Zm}$ from material with defect and without defect. The graphs, Fig. 4, were made with lift-off $s_{1}=1 \mathrm{~mm}$ and $f_{4}=50 \mathrm{kHz}$ and the depths of defect were changed $r_{1}, r_{2}, r_{3}, r_{4}$, $r_{5}$ (as was mentioned in the previous chapter).

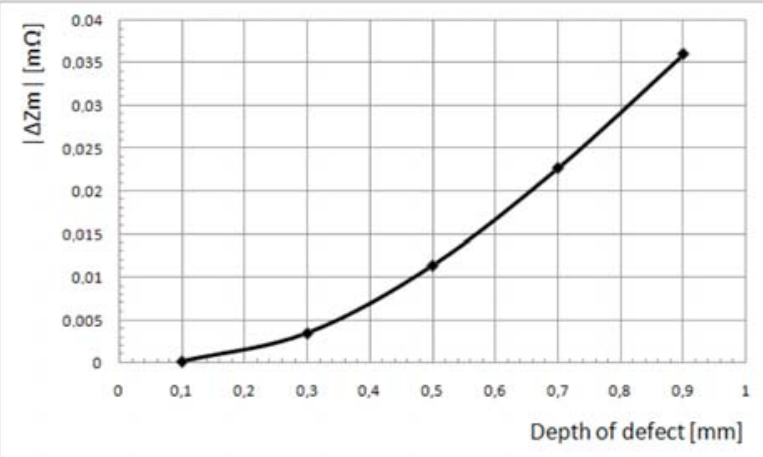

Fig. 4 Dependence of maximum impedance module on defect depth

From the obtained results in graphic form, Fig. 4, showing dependence of maximum impedance module $\left|\Delta Z_{m}\right|$ on the defect depth we can see that with increasing depth of defect also the maximum of impedance module is rising. From the different values of the $\left|\Delta Z_{m}\right|$ it is possible to determine the depth of defect. The points on the waveform for individual depths of defects are wellseparated.

The following obtained results, Fig. 5 were calculated with depth of defect $r_{3}=0.5 \mathrm{~mm}$, exciting frequency $f_{4}=50 \mathrm{kHz}$ and the lift-off was changed $s_{1}=1 \mathrm{~mm}, s_{2}=2 \mathrm{~mm}, s_{3}=3 \mathrm{~mm}$ and $s_{4}=5 \mathrm{~mm}$.

As can be seen from the Fig. 5, with increasing lift-off the $\left|\Delta Z_{m}\right|$ is decreasing. From the waveform, it is evident that the $\left|\Delta Z_{m}\right|$ fall to zero-value. From these results it is possible to determine the lift-off value which is necessary for the sufficient information value of the detected signal. The inductive component is domi-

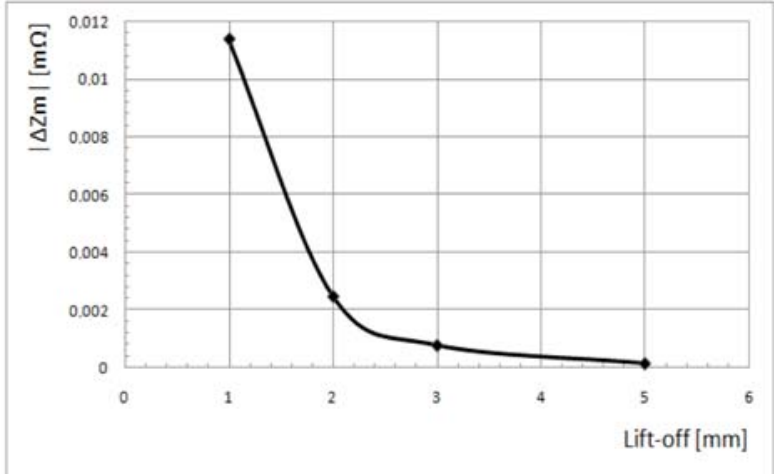

Fig. 5 Dependence of maximum impedance module on lift-off

nant in the probe response signal and thus the dependence of the impedance phase does not show almost any differences for both results graph.

Regarding to these results and to the design of the valve the simulation of the heart valve evaluation for detection of SLF of outlet strut was performed. Because of the complicated design of the valve which does not enable to make a scan of the outlet strut the sweep frequency technique was chosen. According to Fig. 4, the simulations for different values of depth of defect were realized. The choice of the defect depth $r_{5}=0.9 \mathrm{~mm}$ was given by largest difference between gained response signals. Results presented in Fig. 6 were performed for depth of defect $r_{5}=0.9 \mathrm{~mm}$ (it represents SLF) and for lift-off $s_{1}=1 \mathrm{~mm}$. During the simulation the exciting frequency was changed from $f=10 \mathrm{kHz}$ to $f=500 \mathrm{kHz}$ (it represents the frequency sweep with discrete value of frequency). Fig. 6 shows the results of the simulation where the signal from SLF and IOS (intact outlet strut) represents two different waveforms.

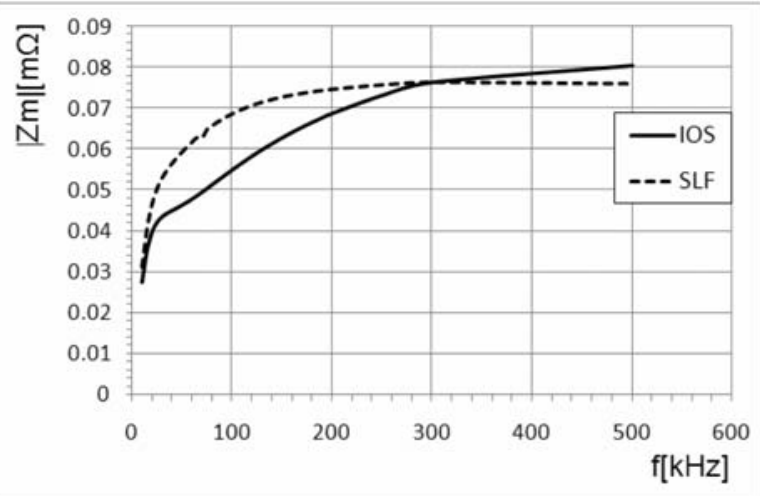

Fig. 6 Dependence of maximum impedance amplitude on frequency

The results show that the detected signal is different for the IOS and SLF valve because of different eddy current flow. This demonstrates that a SLF can be detected by the measuring of the detected signal variation in dependence on frequency. As can be 
seen from Fig. 6, the difference in the dependences between the IOS response signal and the SLF response signal is largest in a frequency range from $70 \mathrm{kHz}$ up to $150 \mathrm{kHz}$.

\section{The ECT with Helmoltz Coils Results}

Fig. 3 shows the simulation results of the electromagnetic system with connected the ECT method with Helmholtz coils shows. The obtained results, Fig. 7, were performed for lift-off $s_{1}=1 \mathrm{~mm}$ and the excitation frequency of the Helmholtz coils was changed from $10 \mathrm{kHz}$ to $500 \mathrm{kHz}$.

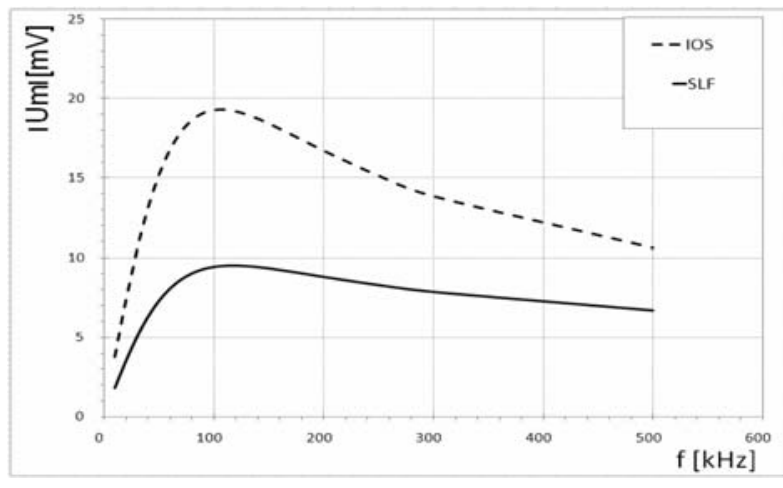

Fig. 7 Dependence of the induced voltage amplitude on frequency
The results, Fig. 7, show that in the vicinity of the outlet strut, the magnetic field perturbations are different for the SLF and IOS heart valve. As can be seen, the magnetic field generated by Helmholtz coils and also the $U_{m}$ response signal which was detected by detection coil are of a higher intensity. These results demonstrate that a SLF can be detected by measuring of the magnetic field changes.

\section{Conclusion}

This paper deals with two electro- magnetic techniques for detection of the SLF in BSCC heart valve. The numerical simulations results confirm that these methods are useful for this purpose. Both methods were presented for both surface and sub-surface cracks detection in conductive materials of the outlet strut. Material defects that can be present in BSCC prosthetic replacement were simulated and the obtained results were discussed. The main inspection parameters such as lift-off, depth of the defect and frequency of the driving coil signal were inspected and analyzed regarding to the coil impedance changes.

\section{Acknowledgement}

This work was supported by the Slovak Research and Development Agency under the contract No. APVV-0194-07. This work was supported by grant of the Slovak Grant Agency VEGA, project No. $1 / 0308 / 08$

\section{References}

[1] VAN NEER, P.: The Bjork-Shiley valve: Detecting Broken Struts Using Standards Diagnostic Ultrasound Instruments, MSc Thesis, 2005

[2] BRAUER, H., ZIOLKOWSKI, M.: Eddy Current Testing of Metallic Sheet with Defects Using Force Measurements, Serbian J. of Electrical Eng., Vol.5, No.1, May, pp. 11-20

[3] CHAN SHIU C., YUE LI, UDPA LALITA, UDPA SATISH S.: Electromagnetic Techniques for Detecting Strut Failures in Artificial Heart Valve, Electromagnetic Nondestructive Evaluation, Vol. 26, Studies in Applied Electromagnetics and Mechanics, G. Dobmann, ISBN 1- 58603-594-0

[4] STRAPACOVA, T., CAPOVA, K.: Influence of Selected Parameters on Detection of Heart Valves Outlet Strut Fractures Using ECT Method, Int'1 Conference AMTEE 2009, Cheb, ISBN 978-80-7043-821-3.

[5] JANOUSEK, L., MAREK, T., GOMBARSKA, D.: Eddy Current Non-destructive Testing of Conductive Materials, Communications - Scientific Letters of the University of Zilina, No. 8, 2006, pp. 29-33, ISSN 1335-4205. 